\title{
Allergen-induced tRNA halves repress focal adhesion of airway smooth muscle cells
}

Megumi Shigematsu ${ }^{1, \dagger}$, Kamlesh Pawar ${ }^{1, \dagger}$, Takuya Kawamura ${ }^{1}$, Sushrut D. Shah ${ }^{2}$, Deepak A. Deshpande $^{2, *}$, and Yohei Kirino ${ }^{1, *}$

${ }^{1}$ Computational Medicine Center, ${ }^{2}$ Center for Translational Medicine, Sidney Kimmel Medical College, Thomas Jefferson University, Philadelphia, Pennsylvania 19107, USA

${ }^{\dagger}$ These authors contributed equally to the work as the first authors.

*Correspondence:

Deepak Deshpande, Ph.D.

1020 Locust Street, Philadelphia, PA 19107, USA

Tel: 215-955-3305

Email: Deepak.Deshpande@jefferson.edu

Yohei Kirino, Ph.D.

1020 Locust Street, Philadelphia, PA 19107, USA

Tel: 215-503-8648

Email: Yohei.Kirino@jefferson.edu 


\begin{abstract}
In the pathogenesis of asthma, inflammatory mediators cause structural and functional changes in resident airway cells including airway smooth muscle (ASM) cells. Here, we report that intranasal treatment of mice with house dust mite (HDM) highly upregulated the expression of tRNA half molecules in asthmatic lungs. The 5'-tRNA haves contain a 2',3'-cyclic phosphate (cP) and thus belong to cP-containing RNAs (cP-RNAs). Capturing the whole cP-RNA transcriptome using cP-RNA-seq revealed the global upregulation of not only tRNA halves but also the cP-RNAs derived from mRNAs and rRNAs. Our investigation of the biological significance of the major upregulated 5'-tRNA half species in human primary ASM cells revealed that the 5'-tRNA halves repress autophosphorylation at Tyr416 of Src protein kinase, a key regulator of the cellular focal adhesion pathway, leading to reduction of ASM cellular focal adhesion. These results assign a novel role as a protein modification modulator to tRNA half molecules and unveil a tRNA-engaged pathway in the molecular pathogenesis of asthma.
\end{abstract}




\section{Introduction}

Asthma is a common chronic inflammatory disease of the lung that affects more than 300 million people globally with a rising prevalence $[1,2]$. The pathogenesis of asthma can be broadly categorized into allergen-induced airway inflammation, remodeling, hyperresponsiveness, and mucus hypersecretion [3-7]. These phenomena involve interplay between infiltrated inflammatory cells and resident airway cells including airway smooth muscle (ASM) cells. The mediators released during inflammation (e.g., cytokines, chemokines, growth factors, matrix proteins) play a pivotal role in structural and functional changes of airway cells. The signaling pathways elicited by these mediators culminate in the activation of transcription factors, and numerous studies have established the role of transcriptional factors during inflammation [8-10]. Following transcription, gene expression can be further regulated at post-transcriptional levels, and short non-coding RNAs (ncRNAs), such as microRNAs (miRNAs), have evolved as one of the key post-transcriptional regulators of gene expression. Indeed, altered expression profiles of miRNAs have been implicated in molecular pathogenesis of asthma $[11,12]$. However, the expressional regulation and functional role of other short ncRNA species in asthma have not been well explored.

Although tRNAs are best known as fundamental adapter components of the translational machinery, recent studies have shown that they are also a source for short ncRNAs [13-19]. In many organisms, specific ncRNAs are generated from mature tRNAs or their precursors, which usually does not affect mature tRNA pools. Instead, the generated tRNA-derived ncRNAs themselves are expressed as functional molecules, rather than degradation products, and play important roles in various biological processes beyond translation. tRNA-derived ncRNAs can be classified into two groups: tRNA halves and tRNA-derived fragments (tRFs). tRNA halves 
range either from the $5^{\prime}$-end to the anticodon-loop (5'-tRNA half) or from the anticodon-loop to the 3 '-end (3'-tRNA half) of a mature tRNA, while tRFs are generally shorter than tRNA halves and are derived from the 5'-part (5'-tRF), 3'-part (3'-tRF), or wholly-internal part (i-tRF) of a tRNA. In mammalian cells, tRNA halves are generated from angiogenin (ANG)-mediated anticodon cleavage of tRNAs, which is triggered by stress and sex hormone signaling pathways [20-22]. The generated tRNA halves have been shown to regulate translation, promote stress granule formation, and promote cell proliferation, and they are implicated in cancers and neurodegenerative diseases [22-28].

Despite their demonstrated functionality, research on tRNA half molecules is still limited; information regarding expression profiles of tRNA halves and their regulation remains elusive. This elusiveness could partly result from technical difficulties related to sequencing the tRNA halves. ANG-generated 5'- and 3'-tRNA halves contain a $2^{\prime}, 3^{\prime}$-cyclic phosphate (cP) or an amino acid at their 3 '-end [22], respectively, which prevents amplification and sequencing of tRNA halves by standard RNA-seq [29]. To resolve the issue, we developed "cP-RNA-seq," which is able to specifically sequence cP-containing RNAs (cP-RNAs) [22, 30, 31]. Our recent genome-wide analyses of short cP-RNA transcriptomes using cP-RNA-seq identified numerous novel cP-RNA species expressed in mouse lungs [32]. The mouse lungs abundantly express not only 5'-tRNA halves but also the cP-RNAs derived from mRNAs and rRNAs [32].

Although the expression of tRNA halves are regulated by various biological situations (e.g., stress, sex hormones), how the allergic reaction and inflammation affect the expression of tRNA halves and other mRNA-/rRNA-derived cP-RNAs remains to be investigated in detail. Here we investigated the expressional changes of 5'-tRNA halves and other cP-RNA species induced by house dust mite (HDM) inhalation in mice, revealing significant upregulation of cP-RNAs in 
the asthmatic lungs. Our elucidation of the biological significance of the major upregulated 5'tRNA half species in human ASM cells further revealed the involvement of 5'-tRNA halves in cellular focal adhesion. These results unveil a novel, tRNA/cP-RNA-engaged pathway in the molecular pathogenesis of asthma. 


\section{Results}

\section{HDM challenge upregulates the expression of tRNA halves and cP-RNAs in mouse lung}

Because 5'-tRNA ${ }^{\mathrm{LysCUU}}$ half was one of the most abundant tRNA halves expressed in mouse lungs [32], exploration of the involvement of tRNA halves in asthma pathogenesis began with the analyses of tRNA ${ }^{\text {LysCUU }}$ halves. Mice were treated intranasally with HDM (or with PBS: control) for 3 weeks and RNAs extracted from their lungs were subjected to northern blot analysis for the expression of $5^{\prime}$ - and $3^{\prime}$-tRNA ${ }^{\text {LysCUU }}$ halves. While the levels of mature tRNA ${ }^{\text {LysCUU }}$ were unchanged, the levels of tRNA halves were significantly upregulated in lungs from HDM-challenged mice compared to control mice (Fig. 1A), suggesting that HDM challenge and resultant allergic inflammation induced the expression of tRNA halves in lungs.

5'-tRNA halves belong to cP-RNAs $[22,31]$, and cP-RNAs are produced not only from tRNAs but also from mRNAs and rRNAs in mouse lungs [32]. Staining of total RNAs revealed HDM-induced accumulation of short RNAs with 30-40 nucleotides (nt) in length (Fig. 1B) that are expected to include tRNA halves and other short cP-RNAs [32]. To identify the expression profiles of the HDM-induced cP-RNA species, the short RNA regions were gelpurified from total RNAs of control or HDM-challenged mice lungs (each three biological replicates) and then subjected to cP-RNA-seq [22, 30]. The method successfully amplified 140-160-bp cDNA products (inserted sequences without adapters were estimated to be $\sim 22-$ 42 bp) whose dependency on T4 PNK treatment indicates that, as expected, the amplified bands were derived from cP-RNAs (Fig. 1C). Consistent with the upregulated expression of tRNA halves and short RNA species by HDM challenge (Fig. 1A, 1B), cP-RNA-seq amplified more abundant cDNAs from the HDM-challenged mice compared to the control mice (Fig. 1C). 
These results collectively suggest that HDM challenge enhances the accumulation of tRNA halves and other cP-RNA species in the mouse lung.

\section{Expression of 5'-tRNA halves and other tRNA-derived cP-RNAs is enhanced by HDM challenge}

Illumina sequencing of the amplified bands by cP-RNA-seq procedure yielded approximately 26-34 million raw reads, of which $>75 \%-87 \%$ were extracted as the cP-RNA reads with a length of 20-45 nt (Table S1). Mapping of the cP-RNA reads to the mouse ncRNAs and genome revealed that not only tRNAs but also mRNAs and rRNAs serve as abundant substrates for cP-RNA production, as reported previously [32]. Read lengths of the extracted cP-RNAs similarly showed broad distribution patterns in control and HDMchallenged mice (Fig. S1).

While the 5'-tRNA half is the major subgroup of tRNA-derived cP-RNAs, the reads of other subgroups (i.e., 3'-tRNA half and 5'-, 3'-, and i-tRF) were also present in cP-RNA libraries. HDM-induced upregulation of cP-RNAs (Fig. 1C) and linear distribution of the subgroups between control and HDM-challenged mice (Fig. 2A) suggested the HDM-induced upregulation of all subgroups of tRNA-derived cP-RNAs. Considering that the mouse genome encodes 55 cyto tRNA isoacceptors with different anticodon sequences [33], the identified 5'tRNA halves in control and HDM-challenged mice were similarly derived from a rather focused subset of tRNAs, such as cyto tRNA ${ }^{\text {GlyGCC }}$, tRNA ${ }^{\text {LysCUU }}$, tRNA ${ }^{\text {GluCUC }}$, tRNA ${ }^{\text {iMetCAU }}$, and tRNA $^{\text {ValCAC }}$, which account for $>88 \%$ of the identified 5'-tRNA halves (Fig. 2B). Among the five most abundant $5^{\prime}$-tRNA half species, the ratio of $5^{\prime}$-tRNA ${ }^{\mathrm{ValCAC}}$ half was specifically decreased in HDM-challenged mice (Fig. 2B), implying that the HDM challenge changed the 
tRNA cleavage preferences. The 3'-terminal nucleotides of the 5'-tRNA halves had a strongly biased composition in being pyrimidines $(\mathrm{C}>\mathrm{U})$ (Fig. 2C). The 3'-terminal nucleotides of 5'tRNA ${ }^{\text {GlyGCC }}$ half and 5'-tRNA ${ }^{\text {ValCAC }}$ half in HDM-challenged mice possessed more cytidine and less uridine compared to those in control mice, implying preferential RNA cleavage at cytidine upon HDM challenge.

To validate the expression and quantity of tRNA-derived cP-RNAs, we focused on the top three abundant species: the 5'-tRNA ${ }^{\text {GlyGCC }}$ half, 5'-tRNA ${ }^{\text {LysCUU }}$ half, and 5'-tRNA ${ }^{\text {GluCUC }}$ half (Fig. 2A, 2B, S2A). We quantified the 5'-tRNA ${ }^{\text {GlyGCC }}$ half and $5^{\prime}$-tRNA ${ }^{\text {LysCUU }}$ half by using a specific TaqMan RT-qPCR established in our previous study [32], and analyzed the expression of 5'-tRNA ${ }^{\text {GluCUC }}$ by northern blot. In TaqMan RT-qPCR, both the 5'-tRNA GlyGCC half and 5'tRNA $^{\text {LysCUU }}$ half were significantly upregulated (20- and 12-fold more on average, respectively) in the lungs of the HDM-challenged mice compared to those of the control mice (Fig. 2D). The upregulation of 5'-tRNA GluCUC by HDM challenge was further confirmed by northern blot analysis (Fig. 2E). Collectively, these results revealed that the HDM challenge significantly enhances the expression of 5'-tRNA halves and other tRNA-derived cP-RNAs in the mouse lung.

\section{Expression of mRNA- and rRNA-derived cP-RNAs is also induced by HDM challenge}

Not only tRNAs but also mRNAs and rRNAs serve as major substrate RNAs for cP-RNA generation [32]. Unlike the case of tRNA-derived cP-RNAs, overall expression profiles of mRNA-derived cP-RNAs showed variation between control and HDM-challenged mice (Fig. S3A). Principal component analyses (PCA) using the reads of mRNA-derived cP-RNAs demonstrated separate clusters of control and HDM-challenged samples, reflecting the variation 
between the control and HDM-challenged mice (Fig. S3B). Furthermore, strong nucleotide composition biases were observed at the 5'- and 3'-ends of the mRNA-derived cP-RNAs. The 5'-end and its upstream nucleotide were mostly adenosine and cytosine, while the 3 '-end and its downstream nucleotide were mostly cytosine and adenosine (Fig. 3A), suggesting that mRNA-derived cP-RNAs are generated by the specific RNA cleavage between cytosine and adenosine. rRNA-derived cP-RNAs also revealed the prominent "CA" nucleotide biases at their 5'- and 3'-terminal regions (Fig. 3A). The "CA" biases became stronger in HDM-challenged mice compared to control mice (Fig. 3A), indicating that HDM challenge especially activates 5'-CA-3' cleavage for generation of mRNA- and rRNA-derived cP-RNAs.

cP-RNAs were derived from many different regions throughout the substrate mRNAs and rRNAs, and relative abundance of specific cP-RNAs were different between control and HDMchallenged mice. For example, in Ft11 mRNA, the cP-RNA generated from nucleotide position (np) 796-820, termed cPR-Ftl1, showed higher relative abundance in HDM-challenged mice compared to control mice (Fig. 3B). Although there are 62 "CA" sequences (potential cleavage sites) throughout Ftl1 mRNAs, not all "CA" sequences were cleaved for cP-RNA production, and not all of the cP-RNA-generating regions were distributed identically between control and HDM-challenged mice, suggesting the presence of preferential sites actively cleaved upon HDM challenge. The preferential cleavage of specific sequences upon HDM challenge matches unidentical expression profiles of mRNA-derived cP-RNA species between control and HDMchallenged mice shown in Fig. S3A-B.

To validate the expression of these cP-RNAs, we focused on the three abundant mRNAderived cP-RNAs from Ftl1, Sftpa1, and Rpl12 mRNAs (termed cPR-Ftl1, cPR-Sftpa1, and cPR-Rpl12, respectively) and two abundant rRNA-derived cP-RNAs from $18 \mathrm{~S}$ and 28S rRNAs 
(termed cPR-18S and cPR-28S, respectively) (Fig. 3B). The cleavage sites for production of these cP-RNAs commonly reside in the loop regions of substrate mRNAs (Fig. S2B-C), possibly because the regions are highly accessible to cP-RNA-generating endoribonuclease. To confirm the presence of a cP at 3 '-end of the three species, mouse lung total RNA was treated with calf intestinal phosphatase (CIP; removes 3'-P but not a cP) or T4 polynucleotide kinase (T4 PNK; removes 3'-P/cP), and efficiency of the ligations between each target RNA and a 3'adapter was examined by TaqMan RT-qPCR, as described in $[31,32]$. As shown in Fig. 3C, all five examined RNAs exhibited the highest amplification signals upon T4 PNK treatment, confirming that they contain a $\mathrm{cP}$ as their major $3^{\prime}$-terminal form. As in the case of 5'-tRNA halves (Fig. 2D), all five examined mRNA- and rRNA-derived cP-RNAs were much more abundantly expressed in the lungs of HDM-challenged mice compared to those of control mice (Fig. 3D), indicating that HDM challenge induces an RNA cleavage at specific 5'-CA-3' sequences, often localized at loop regions of mRNAs and rRNAs, leading to abundant accumulation of mRNA- and rRNA-derived cP-RNAs.

\section{HDM-induced 5'-tRNA halves repress cellular focal adhesion in human ASM cells}

The induction of cP-RNA expression by allergen-induced inflammation in the lungs prompted us to further explore the involvement of those molecules in the regulation of airway cellular functions. Among the upregulated cP-RNA species, we focused on 5'-tRNA halves for functional analyses as their biological significances have been demonstrated in previous studies [22-28]. We selected 5'-tRNA ${ }^{\mathrm{LysCUU}}$ and 5'-tRNA ${ }^{\mathrm{GluCUC}}$ for experimental targets, because they are one of the most abundantly accumulated tRNA-derived cP-RNAs in the lungs of HDMchallenged mice, as was confirmed by sequencings and northern blot analyses (Fig. 1 and 2). 
The two 5'-tRNA halves and a control RNA derived from Renilla Luciferase (R-Luc) were synthesized in vitro and individually transfected into human ASM cells. ASM cells are an integral part of airways and their phenotypic changes significantly contribute to asthma pathogenesis [34-36]. To elucidate the effects of 5'-tRNA halves on ASM cell morphology and function, we utilized the xCELLigence system which can monitor dynamic cellular adherence and spreading by analyzing impedance (show as Cell Index-CI) on the culture plate surface. As shown in Fig. 4A, the transfection of 5'-tRNA halves into ASM cells decreased the CI value throughout the time course. Three independent experiments using different ASM cell cultures derived from different donors showed significant $(\mathrm{p}<0.05) \sim 20 \%$ reduction of CI by the transfection of 5'-tRNA halves in human ASM cells (Fig. 4B). Considering that CI was monitored at an early time point when the cells were re-attaching onto the plate, these results suggest that 5'-tRNA halves negatively regulate cellular focal adhesion.

In an attempt to establish the mechanism by which 5'-tRNA halves regulate ASM cell adhesion, we explored regulation of expression and phosphorylation of focal adhesion-related proteins by 5 'tRNA halves. Focal adhesion kinase (FAK), a master regulator of the focal adhesion pathway, contains a major autophosphorylation site at Tyr397 [37, 38]. The phosphorylation at Try397 (p-Tyr397) of FAK is required to bind to Src, a key downstream kinase in focal adhesion pathway. Src protein autophosphorylates Tyr416 (original numbering in chicken Src; Tyr419 in human [39]), which is required for its kinase activity and therefore for it to proceed to the downstream pathway of focal adhesion $[39,40]$. The other site of the Src protein, Tyr527 (original numbering in chicken Src; Tyr530 in human [39]), is phosphorylated by other kinases such as Csk, which is known to prevent Try416 autophosphorylation $[39,40]$. Western blot analysis revealed that the levels of FAK and its p- 
Tyr397 were unchanged by the transfection of 5'-tRNA halves into human ASM cells (Fig. 4C). Interestingly, p-Tyr416 of Src was significantly decreased in the presence of 5'-tRNA halves although the levels of the total Src and p-Tyr527 of Src were unchanged (Fig. 4C). The reduction of Src p-Tyr416 was also observed in the lungs of HDM-treated mice while the total amount of Src was unchanged (Fig. 4D). These data suggest that the 5'-tRNA halves negatively regulate Src p-Try416. Because p-Try416 is a prerequisite for Src kinase activity [39, 40], the reduction of Src p-Try416 could mechanistically contribute to the 5'-tRNA half-induced reduction of human ASM cellular focal adhesion. 


\section{Discussion}

In this study, we unraveled a previously hidden component of the transcriptome whose expression is induced by HDM challenge in mouse lung. By comprehensively identifying short cP-RNA species expressed in the lungs of control and HDM-challenged mice, and by further experimentally validating and quantifying their expression, we demonstrated that HDMinduced allergic reaction and inflammation promote specific RNA cleavages between cytosine and adenosine that lead to global upregulation of the expression of cP-RNA species derived from tRNAs, mRNAs, and rRNAs in asthmatic lungs (Fig. S5A). Our functional characterization of 5'-tRNA halves revealed a novel role of 5'-tRNA halves in negatively regulating a Src autophosphorylation that is essential for Src kinase activity, which reduces cellular focal adhesion in human ASM cells (Fig. S5B). These results suggest that cP-RNA molecules are not just accumulated as non-functional molecules, such as degradation products, but expressed as significant molecules functionally involved in pulmonary diseases, identifying a novel ncRNA-engaged pathway that contributes to asthma pathogenesis.

The expression and functional role of short and long ncRNAs in airway cells have been investigated in recent studies [41, 42]. High-throughput analysis of RNA samples obtained from clinical samples have revealed the novel species of ncRNAs in lung diseases [41, 43, 44]. Different species of miRNAs are known to be expressed in airway cells, including ASM cells, and play a functional role in the regulation of ASM proliferation, contraction, migration, and secretion [45-47]. Recently, long ncRNAs such as TUG1 and TCF7 have been shown to enhance ASM cell migration and proliferation [48, 49]. Findings from our study identified cPRNAs as another novel class of short ncRNA in airway cells with a potential role in pulmonary physiology and pathology. 
The molecular mechanism(s) behind the induction of cP-RNA expression in asthmatic conditions remains to be elucidated. Earlier studies have shown that stress stimuli and sex hormone signaling pathways induce ANG-catalyzed cleavage of the anticodon-loop of tRNAs, leading to the expression of tRNA halves termed tRNA-derived stress-induced RNAs (tiRNAs) and sex hormone-dependent tRNA-derived RNAs (SHOT-RNAs), respectively [20-22]. In tiRNA biogenesis, decreased levels of RNH1, an ANG inhibitor, trigger tRNA cleavage [50]. Because oxidative stress is induced in asthma and in HDM-challenged mice lung [51-53], the tRNA halves might be upregulated as tiRNAs. Alternatively, various inflammatory mediators (e.g., cytokines, chemokines, growth factors) and the transcription factors activated by those mediators might regulate the expression of ANG and/or other unknown endoribonucleases responsible for tRNA half production. Inflammatory and Th2 cytokines (e.g., TNF $\alpha$, IL-1 $\beta$, IL13, IL-5, IL-4) that are secreted in response to allergen challenge are known to play a central role in the pathogenesis of asthma by modulating cellular functions via gene expression in effector cells including in ASM cells. For example, cytokines regulate expression of ADP ribosyl cyclase (CD38) and myosin light chain kinase, thereby modulating calcium homeostasis and contraction in ASM cells, respectively [54]. The enzyme responsible for the biogenesis of mRNA- and rRNA-derived cP-RNAs is unknown but should possess highly-specific activity for the 5'-CA-3' sequence. Because those cP-RNAs are not evenly produced from overall sequences of substrate RNAs but rather derived from specific loci, the RNA cleavage activity to generate them might be tightly regulated. Further investigations are required to clarify the enzyme and its HDM-induced activity for the generation of cP-RNAs in lungs.

The number of studies reporting the biological significance of tRNA-derived ncRNAs has been increasing, consolidating the role of tRNAs as substrates for functional ncRNAs. Among 
tRNA-derived ncRNAs, 5'-tRNA halves are the molecules whose molecular functions are particularly evident; 5'-tRNA halves have been reported to regulate translation, promote stress granule formation, and promote cell proliferation [22-28]. We here identified a novel role of 5'tRNA halves as negative regulators of protein modification and cellular focal adhesion in ASM cells. A reduction of Src activity induced by reduction in the p-Tyr416 level was identified as a potential mechanism underlying the negative regulation of cellular focal adhesion. Because the expression of dominant-negative Src mutant has been reported to reduce ASM cell migration [55], the lack of Src p-Tyr416 could be sufficient to reduce cellular focal adhesion. Although the p-Tyr527 of Src is known to prevent Src autophosphorylation at Tyr416 [39], the levels of p-Tyr527 were unaffected by 5'-tRNA halves. The in vitro assay failed to observe inhibitory effects of 5'-tRNA halves on Src p-Tyr416 autophosphorylation (Fig. S4), suggesting that the inhibition of Src p-Tyr416 might be indirectly achieved through other protein factors with which 5'-tRNA halves interact. Because some phosphatases, such as PTPN13, can specifically remove p-Tyr416 without affecting p-Tyr527 [56, 57], 5'-tRNA halves might function to promote such phosphatase activity to reduce p-Tyr416. Further studies are needed to clarify the mechanism underlying the function of 5'-tRNA halves in regulating Src phosphorylation and cellular focal adhesion, and to further explore the function of mRNA- and rRNA-derived cP-RNAs.

Airway inflammation results in the excessive proliferation of ASM cells contributing to airway remodeling. ASM cells located in the smooth muscle layer undergo phenotypic modification attaining hyperproliferative phenotype. In addition, studies have shown that ASM cells are capable of migration in response to chemotactic factors released during inflammation or arising from the injured epithelium [58-60], and this migration may contribute to cellular 
hyperplasia [58]. It is possible that myofibroblasts, which have been observed around the airways in the asthmatics, could be smooth muscle cells that have migrated luminally. Similarly, remodeling of blood vessels during atherosclerosis involves the early migration of smooth muscle cells from the media to form a neointima underneath the endothelium [61]. Thus the source of cells for ASM remodeling includes airway infiltration of myofibroblasts or neighboring airway smooth muscle cells in the bundle. It is plausible that the migration of ASM cells is facilitated by decreased cellular adhesion that is induced by $5^{\prime}$-tRNA halves. Further research is required to determine the causal relationship between the 5'-tRNA halves-induced decrease in focal adhesion and airway remodeling.

Finally, ncRNAs have emerged as potential biomarkers for asthma diagnosis and severity. Asthma is a heterogeneous disease with multiple phenotypes, and a "one drug fit all" concept does not work. Therefore, specific biomarkers are necessary in tailoring the prevention methods and therapies. Numerous miRNAs, long ncRNAs, and circulating RNAs have been studied in an attempt to develop them as biomarkers for asthma phenotype and severity. This search for biomarkers has included exploration of ncRNAs in exosomes isolated from peripheral blood samples of asthmatics [62-64]. 5'-tRNA halves and other cP-RNAs may potentially be exploited as biomarkers for asthma because of their abundant expression and their drastic increase in asthmatic conditions. 


\section{Materials and Methods}

\section{Ethics statement}

All mouse experiments were conducted in compliance with the standards and guidelines of the National Institutes of Health $(\mathrm{NIH})$ and were approved by the Institutional Animal Care and Use Committee at Thomas Jefferson University. Human ASM cultures were established using procedures approved by Thomas Jefferson University Institutional Review Board.

\section{Mouse models of allergic asthma}

Intranasal challenge of HDM was performed as described previously [65]. Briefly, 8-weekold female C57BL/6 mice were intranasally challenged 5 days a week for 3 consecutive weeks with $25 \mu \mathrm{g}$ of HDM extract (Dermatophagoides pteronyssinus, Greer Labs) in $35 \mu 1$ saline. At 24 hours following the last challenge, lungs were harvested for analyses.

\section{Human ASM cells}

Human ASM cells were isolated from human tracheae or primary bronchi using enzyme dissociation method and then grown in Ham's F-12 medium with supplements as described previously [66]. Cells in subculture during the second through fifth cell passages were used. For experiments requiring serum starvation, the cells were maintained in Ham's F-12 medium with no serum and supplemented with $1 \%$ insulin transferrin selenium (arresting medium) for 24-72 h. The cells were transfected with $50 \mathrm{nM}$ of each synthetic RNA using RNAiMAX

(Invitrogen). After $6 \mathrm{~h}$, the medium was changed to Ham's F-12 containing platelet-derived growth factors (PDGF). 


\section{RNA isolation and enzymatic treatment}

Total RNAs from frozen tissues were extracted using TRIsure (Bioline) as previously described [32]. To investigate the terminal structures of the identified cP-RNAs, total RNA was treated with CIP (New England Biolabs) and T4 PNK (New England Biolabs) as previously described $[22,31]$.

\section{Northern blot}

Northern blot was performed as previously described $[22,67]$ with the following antisense probes: 5'-tRNA ${ }^{\text {LysCUU }}$ half, 5'- GTCTCATGCTCTACCGACTG-3'; 3'-tRNA ${ }^{\text {LysCUU }}$ half, 5'GCTCGAACCCACGACCCTGAGA-3'; 5'-tRNA ${ }^{\text {GluCUC }}$ half, 5'GCCGAATCCTAACCACTAGAC-3'; and 5S rRNA, 5'- GTTCAGGGTGGTATGGCCGT-3'.

\section{cP-RNA-seq and bioinformatics}

20- to 45-nt RNAs were gel-purified from total RNA of mouse lungs and were subjected to cP-RNA-seq procedure as previously described $[22,30,31]$. The amplified cDNAs were gelpurified and sequenced using the illumina NextSeq 500 at the MetaOmics Core Facility of Sidney Kimmel Cancer Center in Thomas Jefferson University. The cP-RNA-seq libraries contain 26-34 million raw reads (Table S1) and are publically available at NCBI's Sequence Read Archive (accession No. SRR8734323; SRR8734324; SRR8734325; SRR8734326; SRR8734327; and SRR8734328). Bioinformatics analyses were performed as described in [32]. In brief, after trimming of 3'-adapter sequence and selecting 25- to 45-nt reads, we used Rbowtie (1.15.1) for the mappings with one mismatch allowed. The reads were mapped to cyto tRNAs, rRNAs, mitochondrial genome, mRNAs, and whole genome. All of the sequences and 
annotations, except for the mito genome, were derived from the Mus musculus GRCm38 (mm10) genome assembly. For differential expression analysis, the sequences of mRNAderived cP-RNAs were analyzed by DESeq2 [68].

\section{Quantification of cP-RNAs by TaqMan RT-qPCR}

TaqMan RT-qPCR for specific quantification of 5'-tRNA halves and other cP-RNAs was performed as previously described $[22,31,32]$. Briefly, to remove a cP from cP-RNAs, total RNA was treated with T4 PNK, followed by ligation to a 3'-RNA adapter by T4 RNA ligase. Ligated RNA was then subjected to TaqMan RT-qPCR using the One Step PrimeScript RTPCR Kit (Clontech), $200 \mathrm{nM}$ of a TaqMan probe targeting the boundary of the target RNA and 3'-adapter, and forward and reverse primers. Sequences of TaqMan probes and primers are shown in Table S2.

\section{Quantification of mRNA by standard RT-qPCR}

ActB mRNA was quantified by standard RT-qPCR. Briefly, DNase I-treated total RNA was subjected to reverse transcription using RevertAid Reverse Transcriptase (ThermoFisher) and a reverse primer. The synthesized cDNAs were then subjected to PCR using $2 \times q P C R$ Master Mix (Bioland) and forward and reverse primers. Sequences of primers are shown in Table S2.

\section{In vitro RNA synthesis}

The synthetic RNAs used in this study, shown in Table $\mathbf{S 3}$, were synthesized by in vitro reaction as described previously [69]. dsDNA templates were synthesized by using PrimeSTAR GXL DNA Polymerase (TaKaRa Bio) and the primers shown in Table S4. The templates were 
then subjected to in vitro transcription reaction with T7 RNA polymerase (New England Biolabs) at $37^{\circ} \mathrm{C}$ for $4 \mathrm{~h}$. For $5^{\prime}$-tRNA ${ }^{\text {GluCUC }}$ half production, the in vitro synthesized RNA contains the ribozyme sequence to generate a mature 5'-end as described previously [70], and therefore reaction mixture was further incubated for 3 cycles of $90^{\circ} \mathrm{C}$ for 2.5 min and $37^{\circ} \mathrm{C}$ for $15 \mathrm{~min}$, allowing the ribozyme reaction. The synthesized RNAs were gel-purified using denaturing PAGE with single nucleotide resolution.

\section{Cell adhesion assay}

Cell adhesion and spreading were measured by using the xCELLigence real-time cell analysis (RTCA) system (ACEA Biosciences) as described previously [71, 72]. After $24 \mathrm{~h}$ of the RNA transfection, ASM cells were detached by trypsinization and were plated onto E-Plate 16 (ACEA Bioscience) at 12,500 cells/well. The impedance of the plate was then monitored every 1 min for $6 \mathrm{~h}$. Fluctuation of the impedance was calculated as Cell Index over a period of time.

\section{Western blot}

Western blot was performed as described previously [31, 67]. ASM lysates were prepared in RIPA buffer supplemented with cOmplete Protease Inhibitor Cocktail (Sigma) and PhosSTOP (Sigma). The primary antibodies used were: Anti-Src (Cell Signaling Technology), anti-phospho-Src-Tyr416 (Cell Signaling Technology), anti-phospho-Src-Tyr527 (Cell Signaling Technology), anti-FAK (Santa Cruz), anti-phospho-FAK-Tyr397 (Cell Signaling Technology), anti- $\alpha$-smooth muscle Actin (SMA, Abcam), anti-GAPDH (Cell Signaling Technology), and anti-Histone H2A (Cell Signaling Technology). 


\section{Acknowledgements}

We are grateful to the members of our laboratories for helpful discussions. This study was supported by the National Institutes of Health Grant (AI130496 to DD and YK) and in part by the National Institutes of Health Grant (GM106047 to YK, HL137030 to DD), American Cancer Society Research Scholar Grant (RSG-17-059-01-RMC, to YK), W.W. Smith Charitable Trust Grant (C1608, to YK), and Japan Society for the Promotion of Science Postdoctoral Fellowship for Research Abroad (to MS). The authors declare no competing financial interests. 


\section{References}

1. Pawankar, R., et al., Allergic diseases and asthma: a major global health concern. Curr Opin Allergy Clin Immunol, 2012. 12(1): p. 39-41.

2. Masoli, M., et al., The global burden of asthma: executive summary of the GINA Dissemination Committee report. Allergy, 2004. 59(5): p. 469-78.

3. Bousquet, J., et al., Asthma. From bronchoconstriction to airways inflammation and remodeling. Am J Respir Crit Care Med, 2000. 161(5): p. 1720-45.

4. Gerthoffer, W.T., et al., Motility, survival, and proliferation. Compr Physiol, 2012. 2(1): p. 255-81.

5. James, A.L. and S. Wenzel, Clinical relevance of airway remodelling in airway diseases. Eur Respir J, 2007. 30(1): p. 134-55.

6. Ward, C., et al., Reduced airway distensibility, fixed airflow limitation, and airway wall remodeling in asthma. Am J Respir Crit Care Med, 2001. 164(9): p. 1718-21.

7. Holgate, S.T., Pathogenesis of asthma. Clin Exp Allergy, 2008. 38(6): p. 872-97.

8. Lambrecht, B.N. and H. Hammad, The airway epithelium in asthma. Nat Med, 2012. 18(5): p. 684-92.

9. Caramori, G., P. Casolari, and I. Adcock, Role of transcription factors in the pathogenesis of asthma and COPD. Cell Commun Adhes, 2013. 20(1-2): p. 21-40.

10. Erle, D.J. and D. Sheppard, The cell biology of asthma. J Cell Biol, 2014. 205(5): p. 621-31.

11. Deshpande, D.A., et al., MicroRNA Regulation of Airway Inflammation and Airway Smooth Muscle Function: Relevance to Asthma. Drug Dev Res, 2015. 76(6): p. 286-95.

12. Perry, M.M., I.M. Adcock, and K.F. Chung, Role of microRNAs in allergic asthma: present and future. Curr Opin Allergy Clin Immunol, 2015. 15(2): p. 156-62.

13. Phizicky, E.M. and A.K. Hopper, tRNA biology charges to the front. Genes Dev, 2010. 24(17): p. 1832-60.

14. Sobala, A. and G. Hutvagner, Transfer RNA-derived fragments: origins, processing, and functions. Wiley Interdiscip Rev RNA, 2011. 2(6): p. 853-62.

15. Gebetsberger, J. and N. Polacek, Slicing tRNAs to boost functional ncRNA diversity. RNA Biol, 2013. 10(12): p. 1798-806.

16. Anderson, P. and P. Ivanov, tRNA fragments in human health and disease. FEBS Lett, 2014. 588(23): p. 4297-304.

17. Shigematsu, M. and Y. Kirino, tRNA-Derived Short Non-coding RNA as Interacting Partners of Argonaute Proteins. Gene Regul Syst Bio, 2015. 9: p. 27-33.

18. Kumar, P., C. Kuscu, and A. Dutta, Biogenesis and Function of Transfer RNA-Related Fragments (tRFs). Trends Biochem Sci, 2016. 41(8): p. 679-89.

19. Shigematsu, M., S. Honda, and Y. Kirino, Transfer RNA as a source of small functional RNA. J Mol Biol Mol Imag, 2014. 1(2): p. 1-8.

20. $\mathrm{Fu}, \mathrm{H}$., et al., Stress induces tRNA cleavage by angiogenin in mammalian cells. FEBS Lett, 2009. 583(2): p. 437-42.

21. Yamasaki, S., et al., Angiogenin cleaves tRNA and promotes stress-induced translational repression. J Cell Biol, 2009. 185(1): p. 35-42.

22. Honda, S., et al., Sex hormone-dependent tRNA halves enhance cell proliferation in breast and prostate cancers. Proc Natl Acad Sci U S A, 2015. 112(29): p. E3816-25. 
23. Emara, M.M., et al., Angiogenin-induced tRNA-derived stress-induced RNAs promote stress-induced stress granule assembly. J Biol Chem, 2010. 285(14): p. 10959-68.

24. Ivanov, P., et al., Angiogenin-Induced tRNA Fragments Inhibit Translation Initiation. Molecular Cell, 2011. 43(4): p. 613-623.

25. Ivanov, P., et al., G-quadruplex structures contribute to the neuroprotective effects of angiogenin-induced tRNA fragments. Proc Natl Acad Sci U S A, 2014. 111(51): p. 18201-6.

26. Blanco, S., et al., Aberrant methylation of tRNAs links cellular stress to neurodevelopmental disorders. EMBO J, 2014. 33(18): p. 2020-39.

27. Lyons, S.M., et al., YB-1 regulates tiRNA-induced Stress Granule formation but not translational repression. Nucleic Acids Res, 2016. 44(14): p. 6949-60.

28. Dhahbi, J.M., et al., 5' tRNA halves are present as abundant complexes in serum, concentrated in blood cells, and modulated by aging and calorie restriction. BMC Genomics, 2013. 14: p. 298.

29. Shigematsu, M., T. Kawamura, and Y. Kirino, Generation of 2',3'-Cyclic PhosphateContaining RNAs as a Hidden Layer of the Transcriptome. Front Genet, 2018. 9: p. 562.

30. Honda, S., K. Morichika, and Y. Kirino, Selective amplification and sequencing of cyclic phosphate-containing RNAs by the cP-RNA-seq method. Nat Protoc, 2016. 11(3): p. 476-89.

31. Honda, S., et al., Increasing cell density globally enhances the biogenesis of Piwiinteracting RNAs in Bombyx mori germ cells. Sci Rep, 2017. 7(1): p. 4110.

32. Shigematsu, M., et al., Genome-wide identification of short cyclic phosphate-containing RNAs and their regulation in aging. PLoS Genet, in press.

33. Chan, P.P. and T.M. Lowe, GtRNAdb: a database of transfer RNA genes detected in genomic sequence. Nucleic Acids Res, 2009. 37(Database issue): p. D93-7.

34. Munakata, M., Airway remodeling and airway smooth muscle in asthma. Allergol Int, 2006. 55(3): p. 235-43.

35. Doeing, D.C. and J. Solway, Airway smooth muscle in the pathophysiology and treatment of asthma. J Appl Physiol (1985), 2013. 114(7): p. 834-43.

36. Fehrenbach, H., C. Wagner, and M. Wegmann, Airway remodeling in asthma: what really matters. Cell Tissue Res, 2017. 367(3): p. 551-569.

37. Parsons, J.T., Focal adhesion kinase: the first ten years. J Cell Sci, 2003. 116(Pt 8): p. 1409-16.

38. Parsons, J.T., et al., Focal adhesion kinase: targeting adhesion signaling pathways for therapeutic intervention. Clin Cancer Res, 2008. 14(3): p. 627-32.

39. Roskoski, R., Jr., Src protein-tyrosine kinase structure and regulation. Biochem Biophys Res Commun, 2004. 324(4): p. 1155-64.

40. Roskoski, R., Jr., Src kinase regulation by phosphorylation and dephosphorylation. Biochem Biophys Res Commun, 2005. 331(1): p. 1-14.

41. Narozna, B., W. Langwinski, and A. Szczepankiewicz, Non-Coding RNAs in Pediatric Airway Diseases. Genes (Basel), 2017. 8(12).

42. Perry, M.M., et al., Role of non-coding RNAs in maintaining primary airway smooth muscle cells. Respir Res, 2014. 15: p. 58.

43. Vencken, S.F., C.M. Greene, and P.J. McKiernan, Non-coding RNA as lung disease biomarkers. Thorax, 2015. 70(5): p. 501-3. 
44. Mestdagh, P., et al., Non-coding RNAs and respiratory disease. Thorax, 2015. 70(4): p. 388-90.

45. Kuhn, A.R., et al., MicroRNA expression in human airway smooth muscle cells: role of miR-25 in regulation of airway smooth muscle phenotype. Am J Respir Cell Mol Biol, 2010. 42(4): p. 506-13.

46. Dileepan, M., et al., MicroRNA-708 regulates CD38 expression through signaling pathways JNK MAP kinase and PTEN/AKT in human airway smooth muscle cells. Respir Res, 2014. 15: p. 107.

47. Szymczak, I., J. Wieczfinska, and R. Pawliczak, Molecular Background of miRNA Role in Asthma and COPD: An Updated Insight. Biomed Res Int, 2016. 2016: p. 7802521.

48. Lin, J., et al., Long noncoding RNA TUG1 promotes airway smooth muscle cells proliferation and migration via sponging miR-590-5p/FGF1 in asthma. Am J Transl Res, 2019. 11(5): p. 3159-3166.

49. Fan, M., et al., Long non-coding RNA TCF7 contributes to the growth and migration of airway smooth muscle cells in asthma through targeting TIMMDC1/Akt axis. Biochem Biophys Res Commun, 2019. 508(3): p. 749-755.

50. Saikia, M., et al., Genome-wide identification and quantitative analysis of cleaved tRNA fragments induced by cellular stress. J Biol Chem, 2012. 287(51): p. 42708-25.

51. Nadeem, A., et al., Increased oxidative stress and altered levels of antioxidants in asthma. J Allergy Clin Immunol, 2003. 111(1): p. 72-8.

52. Dozor, A.J., The role of oxidative stress in the pathogenesis and treatment of asthma. Ann N Y Acad Sci, 2010. 1203: p. 133-7.

53. Chan, T.K., et al., House dust mite-induced asthma causes oxidative damage and DNA double-strand breaks in the lungs. J Allergy Clin Immunol, 2016. 138(1): p. 84-96 e1.

54. Deshpande, D.A., et al., CD38 in the pathogenesis of allergic airway disease: Potential therapeutic targets. Pharmacol Ther, 2017. 172: p. 116-126.

55. Krymskaya, V.P., et al., Src is necessary and sufficient for human airway smooth muscle cell proliferation and migration. FASEB J, 2005. 19(3): p. 428-30.

56. Palmer, A., et al., EphrinB phosphorylation and reverse signaling: regulation by Src kinases and PTP-BL phosphatase. Mol Cell, 2002. 9(4): p. 725-37.

57. Glondu-Lassis, M., et al., PTPL1/PTPN13 regulates breast cancer cell aggressiveness through direct inactivation of Src kinase. Cancer Res, 2010. 70(12): p. 5116-26.

58. Salter, B., et al., Regulation of human airway smooth muscle cell migration and relevance to asthma. Respir Res, 2017. 18(1): p. 156.

59. Al-Alwan, L.A., et al., Differential roles of CXCL2 and CXCL3 and their receptors in regulating normal and asthmatic airway smooth muscle cell migration. J Immunol, 2013. 191(5): p. 2731-41.

60. Govindaraju, V., et al., Interleukin-8: novel roles in human airway smooth muscle cell contraction and migration. Am J Physiol Cell Physiol, 2006. 291(5): p. C957-65.

61. Lao, K.H., L. Zeng, and Q. Xu, Endothelial and smooth muscle cell transformation in atherosclerosis. Curr Opin Lipidol, 2015. 26(5): p. 449-56.

62. Haj-Salem, I., et al., Fibroblast-derived exosomes promote epithelial cell proliferation through TGF-beta2 signalling pathway in severe asthma. Allergy, 2018. 73(1): p. 178186. 
63. Mortaz, E., et al., Exosomes in Severe Asthma: Update in Their Roles and Potential in Therapy. Biomed Res Int, 2018. 2018: p. 2862187.

64. Canas, J.A., et al., Exosomes: A new approach to asthma pathology. Clin Chim Acta, 2019. 495: p. 139-147.

65. Sharma, P., et al., Bitter Taste Receptor Agonists Mitigate Features of Allergic Asthma in Mice. Sci Rep, 2017. 7: p. 46166.

66. Deshpande, D.A., et al., CD38/cyclic ADP-ribose-mediated Ca2+ signaling contributes to airway smooth muscle hyper-responsiveness. FASEB J, 2003. 17(3): p. 452-4.

67. Honda, S., et al., Mitochondrial protein BmPAPI modulates the length of mature piRNAs. RNA, 2013. 19(10): p. 1405-18.

68. Love, M.I., W. Huber, and S. Anders, Moderated estimation of fold change and dispersion for RNA-seq data with DESeq2. Genome Biol, 2014. 15(12): p. 550.

69. Shigematsu, M. and Y. Kirino, 5'-Terminal nucleotide variations in human cytoplasmic tRNAHisGUG and its 5'-halves. RNA, 2017. 23(2): p. 161-168.

70. Nkuipou-Kenfack, E., et al., The use of urinary proteomics in the assessment of suitability of mouse models for ageing. PLoS One, 2017. 12(2): p. e0166875.

71. Koks, S., et al., Mouse models of ageing and their relevance to disease. Mech Ageing Dev, 2016. 160: p. 41-53.

72. Bilkei-Gorzo, A., Genetic mouse models of brain ageing and Alzheimer's disease. Pharmacol Ther, 2014. 142(2): p. 244-57. 


\section{Figure legends}

\section{Figure 1. HDM-induced upregulation of the expression of tRNA halves and cP-RNAs in} mouse lung

(A) Lung total RNAs from mice challenged with PBS (Control) or HDM were subjected to northern blot analyses for 5'-tRNA ${ }^{\text {LysCUU }}$ half (left) and 3'-tRNA ${ }^{\text {LysCUU }}$ half (right).

(B) Lung total RNAs from control or HDM-challenged mice were developed by denaturing PAGE and stained by SYBR gold. The short RNA regions accumulated by HDM-challenge are shown with a line.

(C) Gel-purified 20-45-nt RNAs from control or HDM-challenged mice (each three biological replicates: \#1-3) were subjected to cP-RNA-seq, which amplified 140-160-bp cDNA products (5'-adapter, $55 \mathrm{bp}$; 3'-adapter, $63 \mathrm{bp}$; and thereby estimated inserted sequences, $22-42 \mathrm{bp}$ ). The cDNAs in the region designated with a line were purified and subjected to Illumina sequencing.

Figure 2. Analyses of tRNA-derived cP-RNAs expressed in the lungs of HDM-challenged mice

(A) Scatter plots of reads per million (RPM) of tRNA-derived RNAs identified by cP-RNA-seq in lungs of control or HDM-challenged mice.

(B) Proportion of the 5'-tRNA half-reads derived from respective cyto tRNA species.

(C) Proportion of the 3'-terminal positions of the 5'-tRNA halves.

(D) Lung total RNAs from control or HDM-challenged mice were subjected to TaqMan RTqPCR for quantification of the 5'-tRNA ${ }^{\text {GlyGCC }}$ half and 5'-tRNA ${ }^{\text {LysCUU }}$ half. ActB mRNA was quantified as the control whose expression is unchanged by HDM challenge. $* * P<0.01$ by $t$ test. 
(E) Lung total RNAs from control or HDM-challenged mice were subjected to northern blot analysis for 5'-tRNA ${ }^{\text {GluCUC }}$.

Figure 3. Analyses of mRNA- and rRNA-derived cP-RNAs expressed in the lungs of HDM-challenged mice

(A) Nucleotide compositions around the 5'- and 3'-ends of mRNA- and rRNA-derived cPRNAs. A dashed line separates upstream (-) and downstream (+) positions for the 5'-and 3'ends, representing the cleavage site that generates mRNA-derived cP-RNAs (the regions outside of cP-RNA-generating regions are colored in grey).

(B) The alignment patterns of cP-RNAs for the indicated mRNA and rRNA substrates. The position of the three focused mRNA-derived cP-RNAs and two focused rRNA-derived cPRNAs are shown.

(C) Lung total RNAs were treated with CIP or T4 PNK (PNK) and subjected to TaqMan RTqPCR for the specific quantification of the indicated cP-RNAs. NT designates a non-treated sample used as a negative control. Averages of three independent experiments with SD values are shown.

(D) Lung total RNAs control or HDM-challenged mice were subjected to TaqMan RT-qPCR for the indicated cP-RNAs. ${ }^{*} P<0.05, * * * P<0.001$ by $t$-test. Although each of the six biological replicates were analyzed, in the quantification of mRNA-derived cP-RNAs, only three (for cPR-Sftpa1/Rp112) or four (for cPR-Ftl1) replicates of control mice showed amplification signals, mostly likely due to their low expression levels in control mice. Because of the lack of the data, $\mathrm{P}$ values are not shown for mRNA-derived cP-RNAs. 


\section{Figure 4. Functional significance of $5^{\prime}$-tRNA halves in cellular focal adhesion}

(A) The adhesion and spreading of ASM cells after transfection of 5'-tRNA GluCUC half (5'GluCUC), 5'-tRNA ${ }^{\text {LysCUU }}$ half (5'-LysCUU), or R-Luc-derived short RNA (R-Luc: control) were monitored by xCELLigence system.

(B) Integrated CI values were obtained from 0-4 h time frame of xCELLigence data. The value of control cells was set as 1 , and relative amounts are indicated. Averages of three independent experiments using three different lines of ASM cells (\#1-3) with SD values are shown.

(C) Total cell lysates from the three different ASM cell lines (\#1-3) transfected with the indicated RNAs were subjected to western blot analyses with the indicated antibodies.

(D) Lysates from lungs of control or HDM-challenged mice were subjected to western blot analyses with the indicated antibodies. The HDM-induced asthmatic condition was confirmed by the increased amounts of $\alpha$-smooth muscle actin (SMA). 

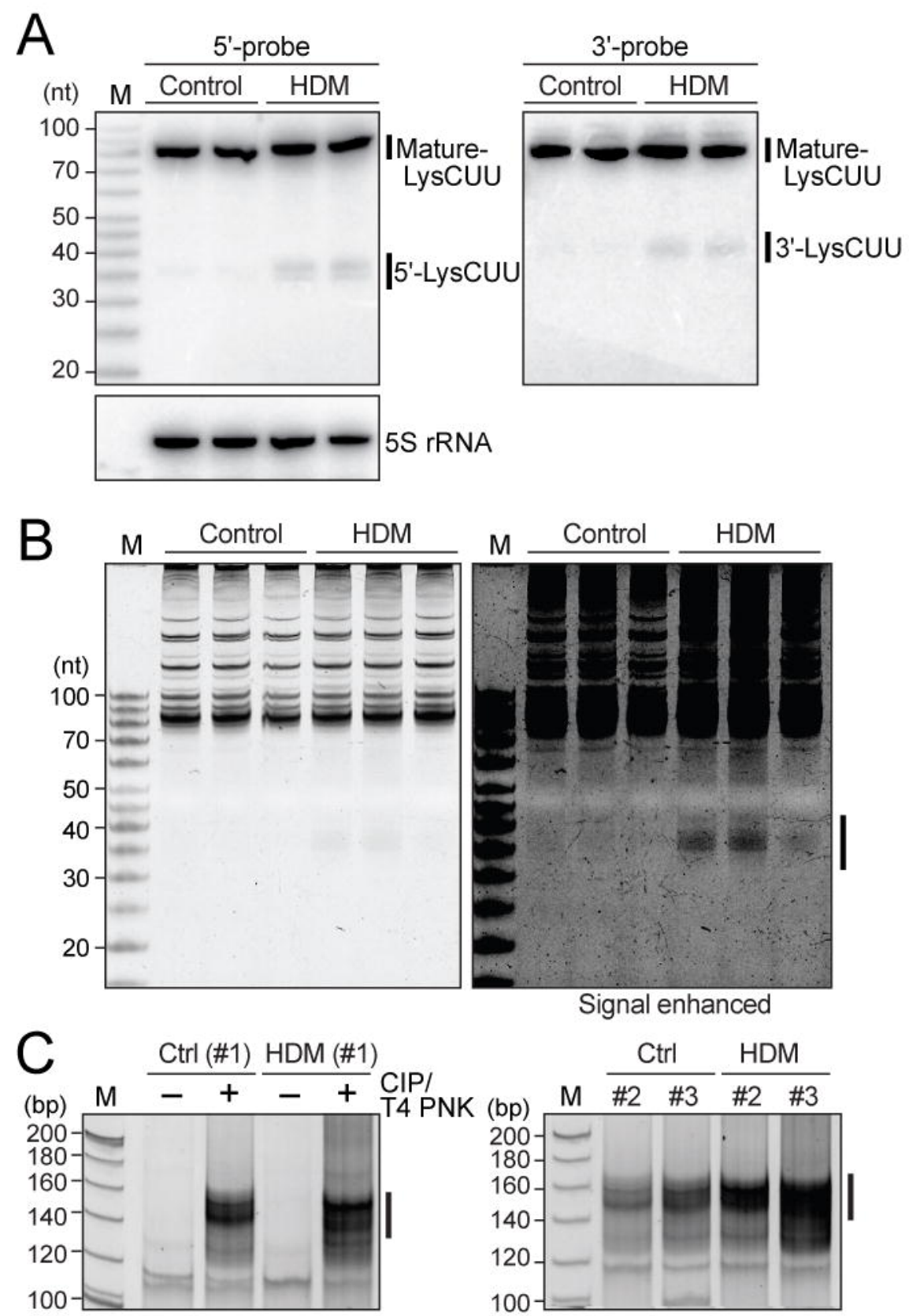

Figure 1 

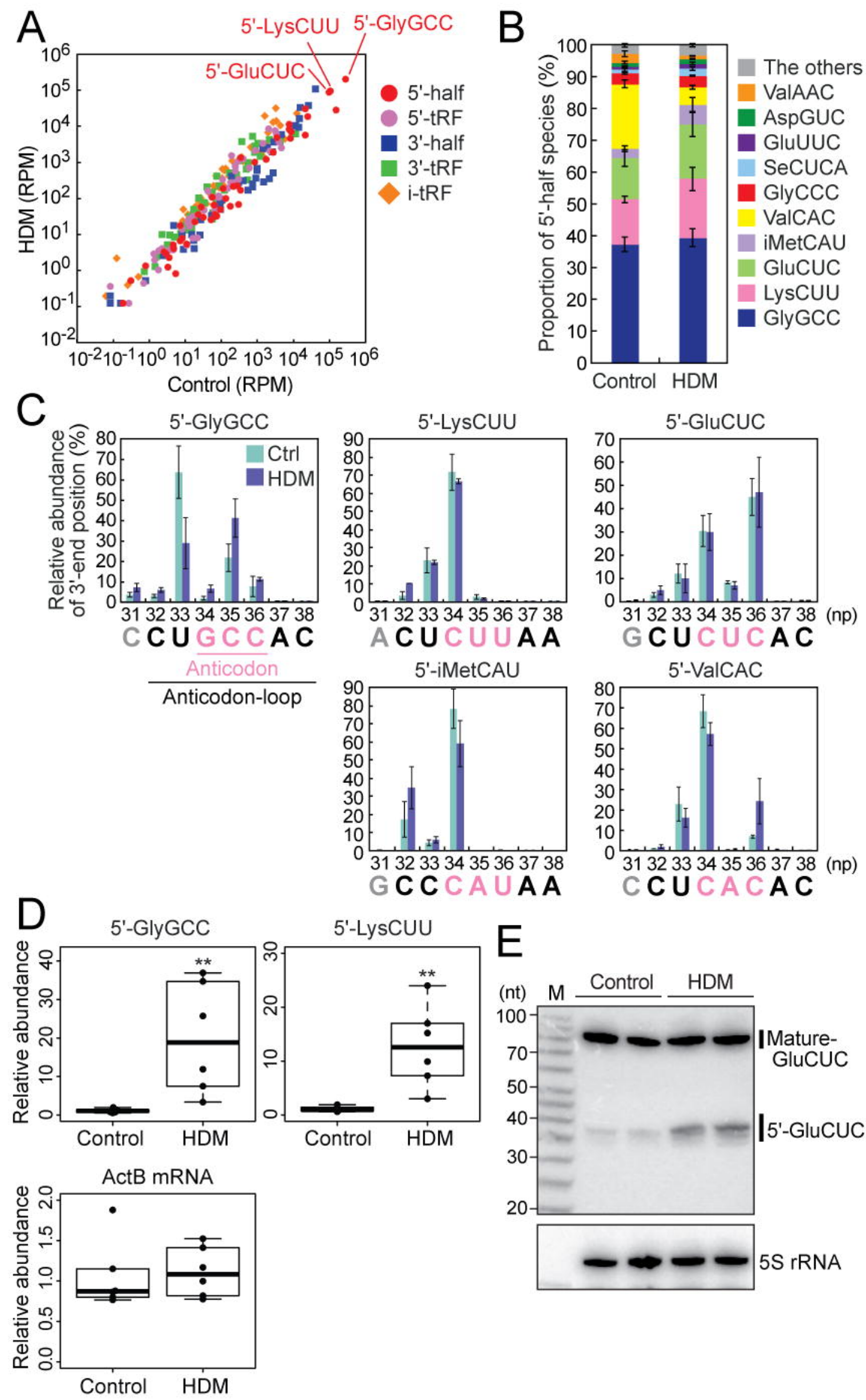
A

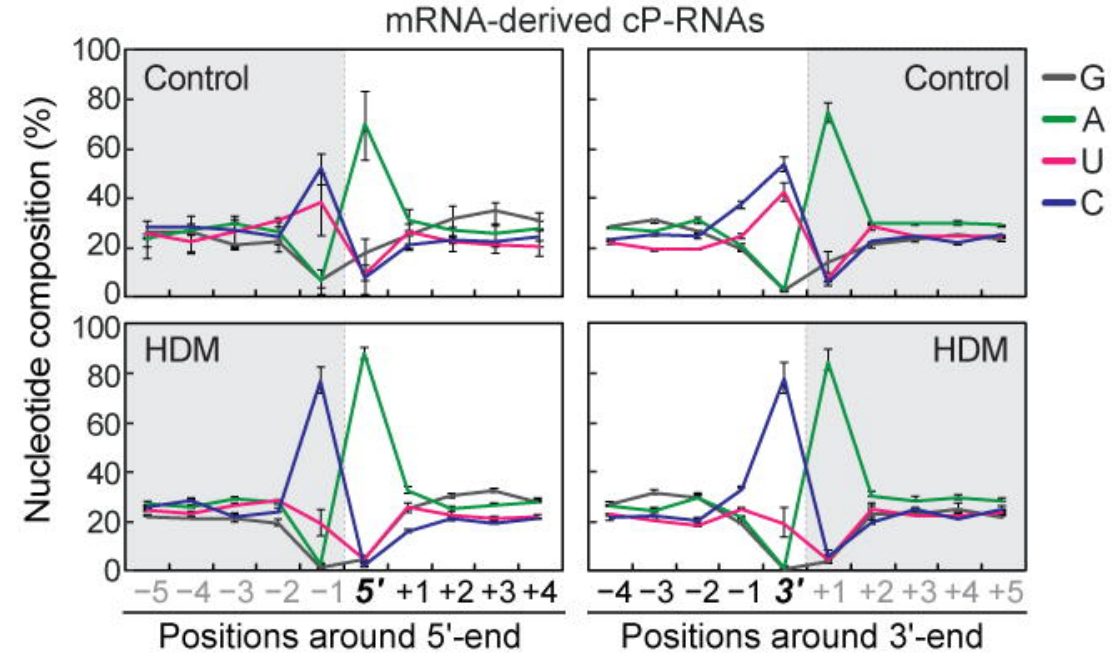

rRNA-derived cP-RNAs

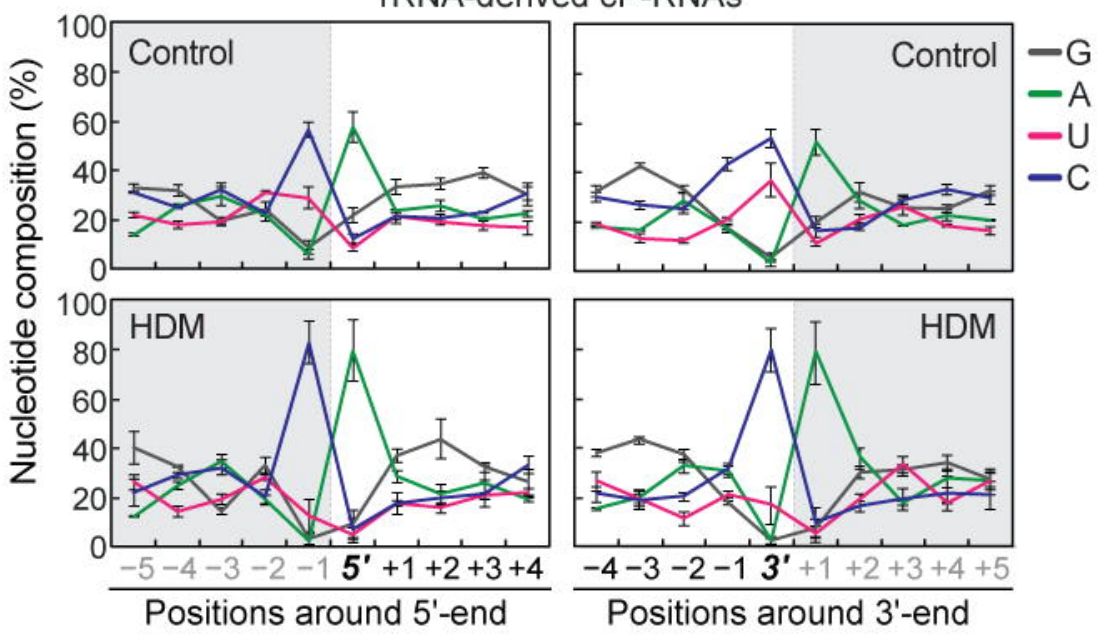

C

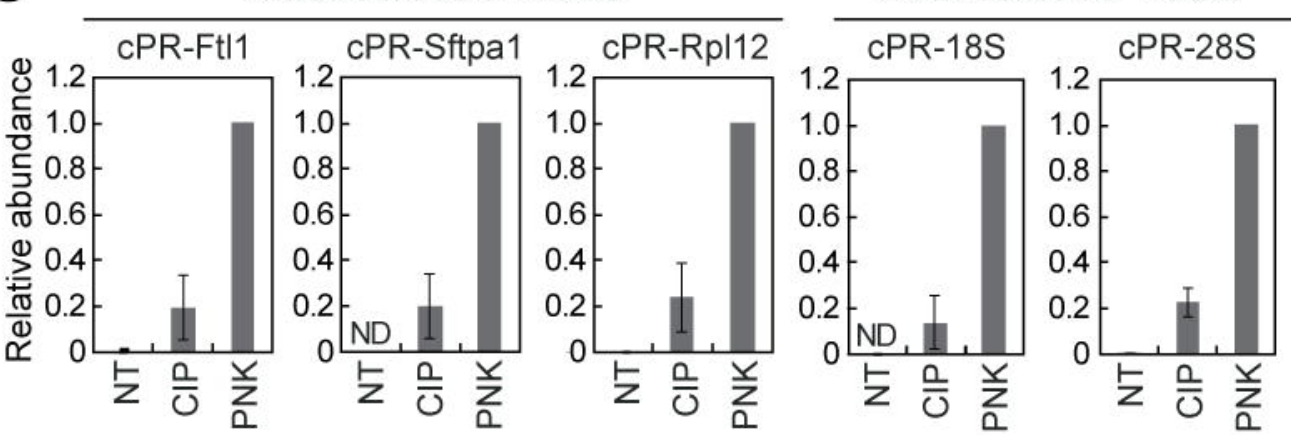

$\mathrm{D}$

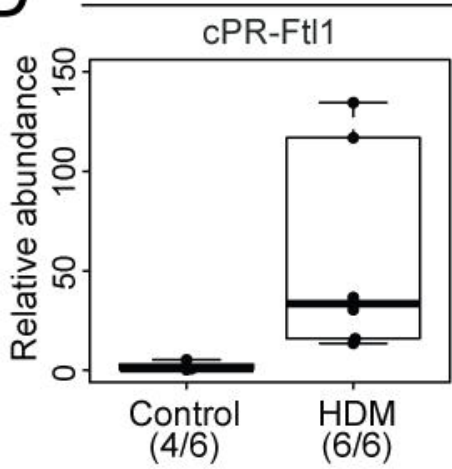

mRNA-derived cP-RNAs
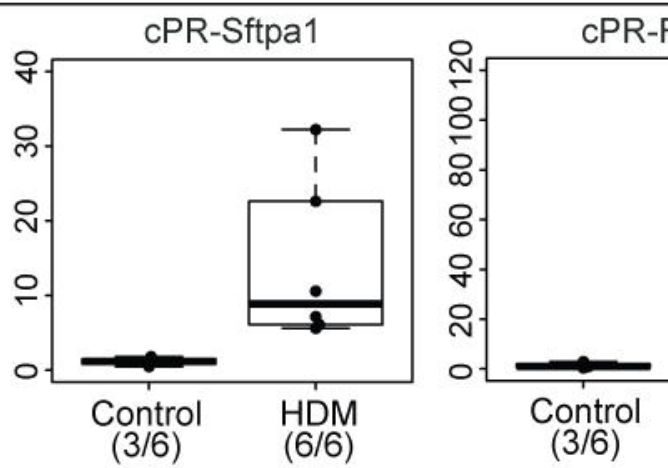

B

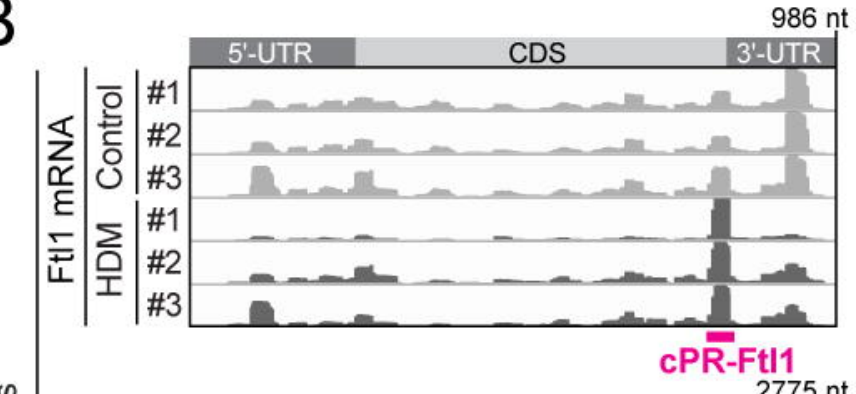

$\sum_{\substack{\infty \\ 1}}^{\infty}$

:

.
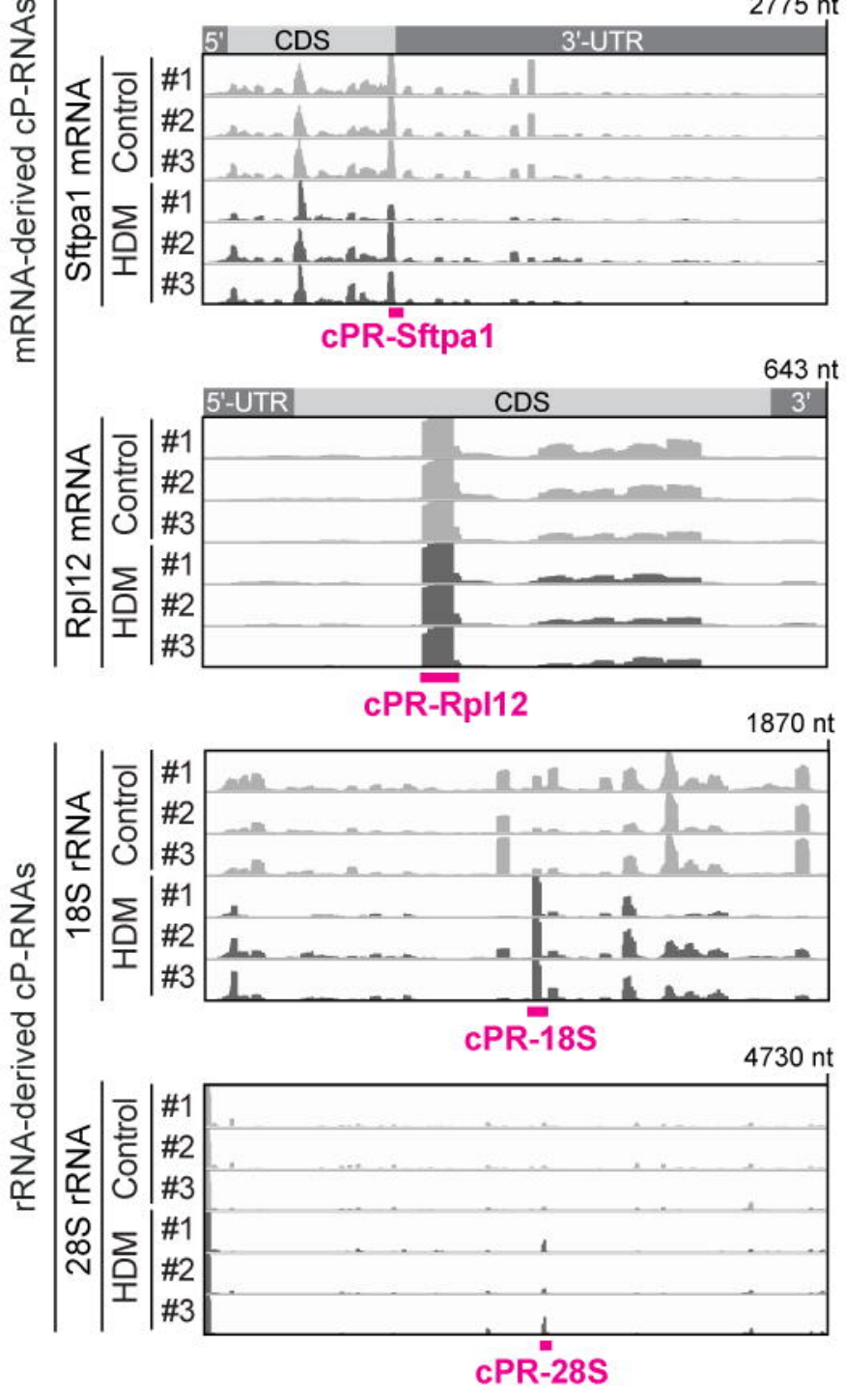

rRNA-derived cP-RNAs

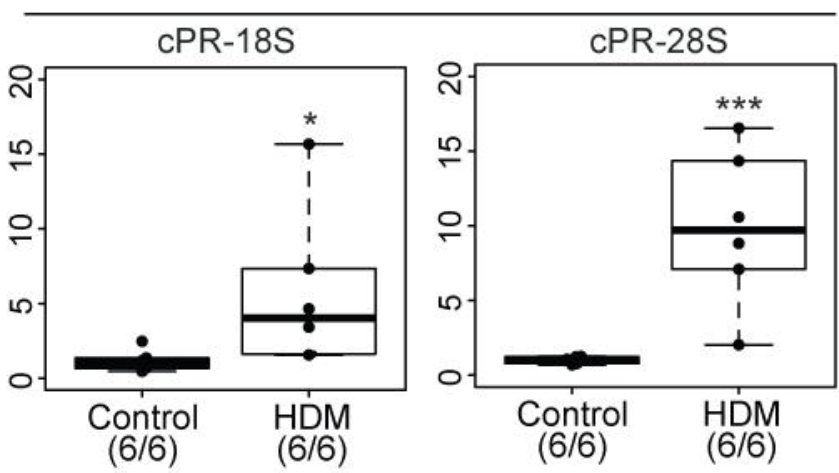


A

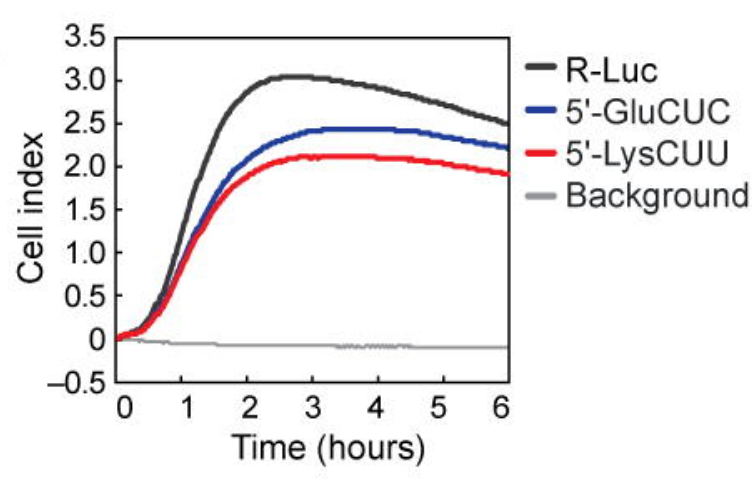

C

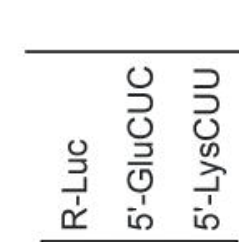

FAK
p-Tyr397

FAK

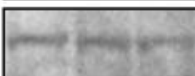
Src
p-Tyr527

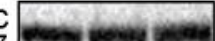

\#1
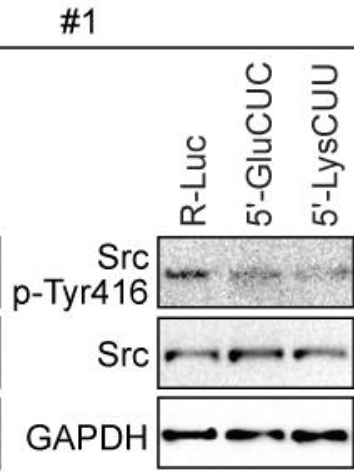

$\mathrm{D}$

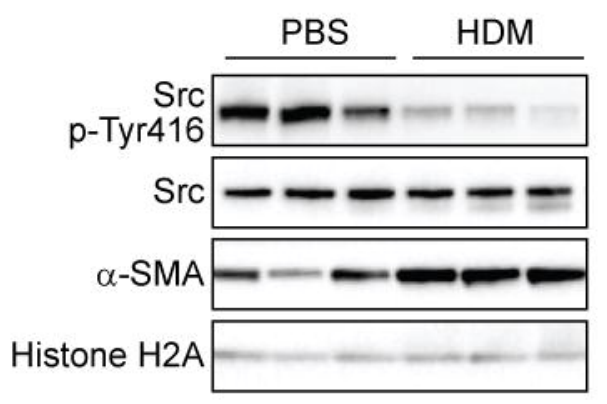

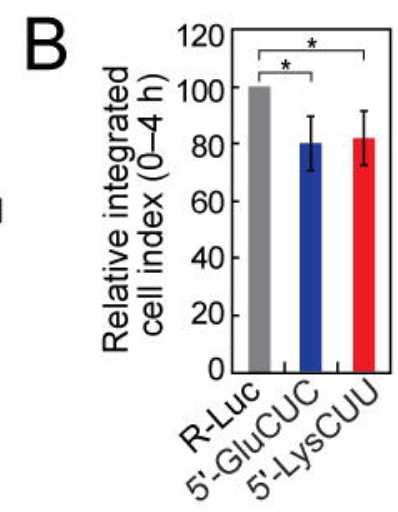

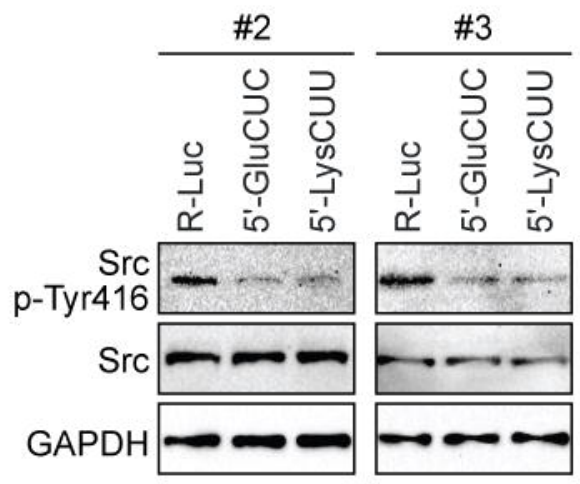

Figure 4 